\title{
Hopf bifurcation from viscous shock waves
}

\author{
Björn Sandstede * $\quad$ Arnd Scheel ${ }^{\dagger}$
}

November 21, 2006

\begin{abstract}
Using spatial dynamics, we prove a Hopf bifurcation theorem for viscous Lax shocks in viscous conservation laws. The bifurcating viscous shocks are unique (up to time and space translation), exponentially localized in space, periodic in time, and their speed satisfies the Rankine-Hugoniot condition. We also prove an "exchange of spectral stability" result for super- and subcritical bifurcations, and outline how our proofs can be extended to cover degenerate, over-, and undercompressive viscous shocks.
\end{abstract}

\section{Introduction}

The purpose of this paper is to study Hopf bifurcation from viscous shock waves. While Hopf bifurcations from equilibria are well understood in ordinary differential equations and in dissipative partial differential equations (PDEs) on bounded domains, a variety of new phenomena and difficulties arise when studying Hopf bifurcations for PDEs on unbounded domains.

In particular, Hopf bifurcations from travelling waves are complicated by the presence of a neutral mode at the origin which is induced by spatial translation. If the essential spectrum of the linearization around the travelling wave is bounded away from the imaginary axis, appropriate center-manifold reductions and equivariant parametrizations as in $[4,6,16]$ show that the bifurcation problem reduces to a standard Hopf bifurcation, and standard results on bifurcation and exchange of stability [2] immediately carry over to this setting [14, §2]; the only effect of the translation mode is an adjustment of the wave speed. When the Hopf instability is caused by essential spectrum that crosses the imaginary axis, a variety of interesting new phenomena can occur, including failure of bifurcation [14] and bifurcation of multiple solution branches $[15, \S 2.3]$. The situation becomes more involved when the instability caused by the essential spectrum is stationary, as the wave will then typically decay only algebraically at onset which leads to significant complications in the analysis $[15, \S 2.1-2.2, \S 3]$.

From the preceding list, one can easily envision yet another scenario in which the complex Hopf eigenvalues belong to the point spectrum, whilst the translation mode is embedded in the continuous spectrum. This situation arises, for instance, when the primary wave is not spatially localized, but the Hopf eigenfunctions are localized: Examples are Hopf bifurcations from coherent structures such as sources and sinks in one spatial dimension, and spiral waves in two dimensions. A model problem in higher space dimensions, but with a space-dependent potential, has recently been analysed in [1]. Viscous shock waves provide another prominent example where the translation mode is embedded into the continuous spectrum. In fact, conservation laws can often be derived formally and rigorously in spatially extended systems where the primary pattern breaks the underlying continuous symmetry $[3,7]$.

In this article, we investigate Hopf bifurcations from viscous shock waves using the spatial-dynamics techniques we developed in [12-14] for Hopf bifurcations from fronts and pulses in reaction-diffusion systems.

*Department of Mathematics, University of Surrey, Guildford, GU2 7XH, UK

${ }^{\dagger}$ School of Mathematics, University of Minnesota, Minneapolis, MN 55455, USA 
Our paper is strongly motivated by recent work of Texier and Zumbrun $[18,19]$ in which they analysed oscillatory instabilities of viscous shocks using delicate estimates for the temporal period map of the linearized semigroup. Texier and Zumbrun proved the existence of a continuous branch of oscillatory viscous shocks with a $1 / x$ decay estimate at spatial infinity. In a personal communication, Zumbrun asked us whether spatial-dynamics techniques can be used to obtain the same or stronger results than those in $[18,19]$. We demonstrate here that the spatial-dynamics approach yields indeed sharper results, while simplifying the analysis and adding geometric insight into the problem: We show that the bifurcating oscillatory shocks are unique, exponentially localized, and depend smoothly on the bifurcation parameter, and we calculate the spectra of the linearization about the bifurcating oscillating shock waves, thereby confirming the expected exchange of stability. Instead of analysing the temporal semigroup whose linearization has essential spectrum up to the imaginary axis, we consider the spatial evolution of temporally periodic functions for which we gain compactness of the resolvent due to the imposed time-periodicity. While this method may appear non-intuitive, it is completely analogous to the usual phase-plane analysis used to prove existence of viscous shocks and to study their stationary bifurcations.

Outline: In $\S 2$, we state our main result on bifurcation and spectral stability of modulated shocks. The bifurcation result is proved in $\S 3$. In $\S 4$, we review the precise characterization of spectra and prove stability and instability in the case of super- and subcritical bifurcations, respectively. We conclude with a discussion of several extensions and generalizations in $\S 5$.

\section{Setup and main results}

Consider the viscous conservation law

$$
u_{t}+f(u)_{y}=u_{y y}, \quad y \in \mathbb{R}, u \in \mathbb{R}^{n}
$$

where $f$ is a smooth flux function. We are interested in viscous shocks $q^{0}\left(y-c^{0} t\right)$ which connect the constant rest states $u_{ \pm}^{0}$ at $y= \pm \infty$ so that

$$
\lim _{x \rightarrow \pm \infty} q^{0}(x)=u_{ \pm}^{0}
$$

Viscous shocks are stationary solutions in the moving reference frame $x=y-c t$ in which (2.1) becomes

$$
u_{t}=\partial_{x}\left[u_{x}+c u-f(u)\right], \quad x \in \mathbb{R}, u \in \mathbb{R}^{n},
$$

and they therefore satisfy the integrated steady-state equation

$$
u_{x}=\left[f(u)-f\left(u_{-}\right)\right]-c\left[u-u_{-}\right],
$$

where the speed $c$ is given necessarily by the Rankine-Hugoniot condition

$$
c=\frac{f_{j}\left(u_{+}\right)-f_{j}\left(u_{-}\right)}{u_{+, j}-u_{-, j}}, \quad j=1, \ldots, n .
$$

In particular, $q^{0}(x)$ lies in the intersection of the unstable manifold $W_{-}^{\mathrm{u}}$ of $u_{-}^{0}$ and stable manifold $W_{+}^{\mathrm{s}}$ of $u_{+}^{0}$ for $(2.3)$.

The most common viscous shocks are Lax shocks for which $u_{ \pm}^{0}$ are hyperbolic equilibria of (2.3) with $\operatorname{dim} W_{-}^{\mathrm{u}}=p+1$ and $\operatorname{dim} W_{+}^{\mathrm{s}}=n-p$ for some $p \in\{0, \ldots, n-1\}$. We assume that the intersection of $W_{-}^{\mathrm{u}}$ and $W_{+}^{\mathrm{s}}$ is transverse along $q^{0}$ and that the Jacobian $f_{u}\left(u_{ \pm}^{0}\right)$ has only real and distinct eigenvalues. If $u_{ \pm}^{\varepsilon}$ are smooth curves that depend on a real parameter $\varepsilon \approx 0$, then we find a smooth family of Lax shocks $q^{\varepsilon}(x)$ with a smooth speed relation $c=c^{\varepsilon}$ given by the Rankine-Hugoniot condition. Since the eigenvalues of $f_{u}(u)$ are the characteristic speeds of propagation at $u$, the condition on the dimension of $W_{ \pm}^{\mathrm{s}, \mathrm{u}}$ merely states that $p+1$ characteristics enter the shock from the left and $n-p$ characteristics enter from the right. 

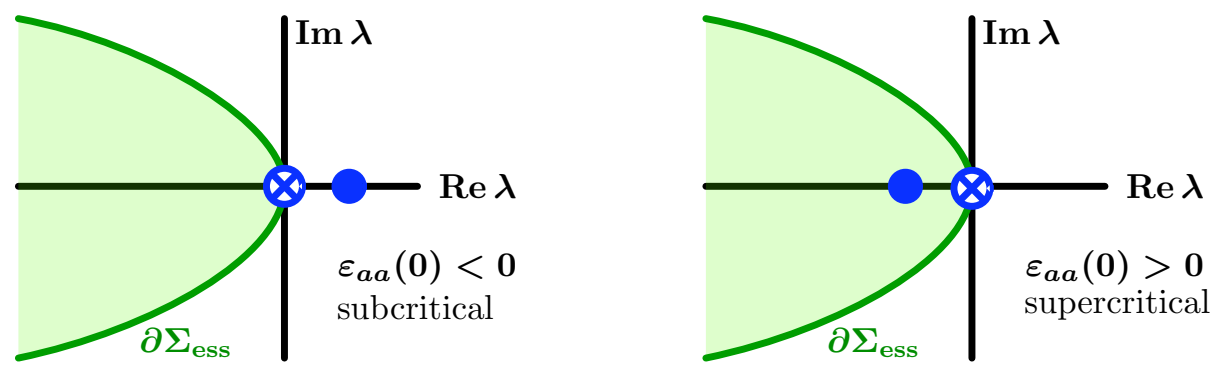

Figure 1: The Floquet spectra of the oscillatory shocks $u^{*}$ from Theorem 1 is shown for $a>0: \lambda=0$ has geometric and algebraic multiplicity two, while the location of the remaining simple Floquet exponent near the origin depends on the sign of $\varepsilon_{a a}(0)$.

We are interested in the scenario where the Lax shocks undergo a Hopf instability upon increasing $\varepsilon$ through zero. We therefore consider the linearization at the shock which is given by the linear operator

$$
\mathcal{L}^{\varepsilon}:=\partial_{x}\left[\partial_{x}+c^{\varepsilon}-f_{u}\left(q^{\varepsilon}(x)\right)\right] .
$$

We consider $\mathcal{L}^{\varepsilon}$ as a closed unbounded operator on $L^{2}\left(\mathbb{R}, \mathbb{R}^{n}\right)$. Its essential spectrum is readily seen to be contained in the closed left half plane, touching the imaginary axis only at the origin with a quadratic tangency. We assume that the point spectrum lies in the open left half plane, bounded away from the imaginary axis, except for an isolated pair $\lambda(\varepsilon)$ and $\overline{\lambda(\varepsilon)}$ of simple complex eigenvalues with

$$
\lambda(0)=\mathrm{i} \omega_{0} \neq 0, \quad \operatorname{Re} \lambda_{\varepsilon}(0)>0 .
$$

Theorem 1 (Bifurcation) Under the above assumptions, there are positive constants $K, \eta$ and $\delta$, and a smooth function

$$
\begin{aligned}
{[0, \delta) } & \longrightarrow \mathcal{C}_{\text {unif }}^{2}\left(S^{1} \times \mathbb{R}, \mathbb{R}^{n}\right) \times \mathbb{R} \times \mathbb{R} \\
a & \longmapsto\left(q^{*}(a), \varepsilon(a), \omega(a)\right)
\end{aligned}
$$

so that $u^{*}(x, t ; a):=q^{*}(x, \omega(a) t ; a)$ satisfies (2.2) with $c=c^{\varepsilon(a)}$ for all $a$,

$$
\left|q^{*}(x, \tau ; a)-u_{ \pm}^{\varepsilon(a)}\right| \leq K \mathrm{e}^{-\eta|x|}, \quad q^{*}(x, \tau ; 0)=q^{0}(x), \quad \omega(0)=\omega_{0},
$$

and $q^{*}(x, \cdot ; a)$ has minimal period $2 \pi$ in $\tau$ for each $a>0$. Furthermore, any non-stationary time-periodic solution $u(x, t)$ of (2.2), which is pointwise close to $q^{0}(x)$ and converges to $u_{ \pm}^{\varepsilon}$ as $x \rightarrow \pm \infty$, is in fact an appropriate space and time translate of $u^{*}$.

Note that $u^{*}$ and $q^{\varepsilon}$ have the same asymptotic rest states and travel with the same (average) wave speed. The above result remains true if $f=f(u ; \varepsilon)$ depends smoothly on the parameter $\varepsilon$.

Spectral stability of the modulated shocks $u^{*}(x, t ; a)$ is determined by the Floquet spectrum

$$
\Sigma=\left\{\lambda \in \mathbb{C} ; \mathrm{e}^{\lambda T} \in \text { spectrum of } \Phi_{T}\right\}
$$

where $T=2 \pi / \omega$ is the temporal period of $u^{*}$, and $\Phi_{t}$ is the evolution operator of the linearization

$$
v_{t}=\partial_{x}\left[v_{x}+c^{\varepsilon(a)} v-f_{u}\left(u^{*}(x, t ; a)\right) v\right]
$$

of (2.2) about $u^{*}$ on $L^{2}$ or $\mathcal{C}_{\text {unif }}^{0}$

Theorem 2 (Stability) Assume that the hypotheses of Theorem 1 are met and that the Evans function associated with $q^{0}$ has a simple zero at the origin (see $\S 4$ for details). If $\varepsilon_{a a}(0) \neq 0$, then the Floquet spectrum $\Sigma$ of the oscillatory shock $u^{*}$ given in Theorem 1 is as indicated in Figure 1.

We refer to $[5, \S 3.4]$ and $[21, \S 9-10]$ for explicit conditions which imply that the Evans function of $q^{0}$ has a simple zero at the origin. 


\section{Existence of modulated viscous shocks}

In this section, we prove Theorem 1.

\subsection{Preparations}

We begin by collecting some properties of the linearization

$$
\mathcal{L}^{\varepsilon}=\partial_{x}\left[\partial_{x}+c^{\varepsilon}-f_{u}\left(q^{\varepsilon}(x)\right)\right]
$$

about the viscous shocks that we need later on. Since we assumed that the Hopf eigenvalues are simple with

$$
\lambda(0)=\mathrm{i} \omega_{0} \neq 0, \quad \operatorname{Re} \lambda_{\varepsilon}(0)>0,
$$

see (2.5), we know that there are nonzero $L^{2}$-functions $v_{j}$ and $\psi_{j}$ for $j=1,2$ that form a basis of the eigenspaces of $\mathcal{L}^{0}$ and its adjoint $\left[\mathcal{L}^{0}\right]^{*}$, respectively, associated with the Hopf eigenvalues $\lambda(0)$ and $\overline{\lambda(0)}$. We can choose these functions so that

$$
\begin{gathered}
\left\langle\psi_{i}, v_{j}\right\rangle_{L^{2}}=\left\langle v_{i}, v_{j}\right\rangle_{L^{2}}=\left\langle\psi_{i}, \psi_{j}\right\rangle_{L^{2}}=\delta_{i j} \\
\mathcal{L}^{0} v_{1}=-\omega_{0} v_{2}, \quad \mathcal{L}^{0} v_{2}=\omega_{0} v_{1} .
\end{gathered}
$$

It is straightforward to check that

$$
\operatorname{Re} \lambda_{\varepsilon}(0)=\left\langle\psi_{1},\left.\partial_{\varepsilon} \mathcal{L}^{\varepsilon}\right|_{\varepsilon=0} v_{1}\right\rangle_{L^{2}}
$$

\subsection{Spatial dynamics}

To find time-periodic solutions of (2.2), we rescale time $\tau:=\omega t$ to get

$$
\omega \partial_{\tau} u+f(u)_{x}-c u_{x}=u_{x x}
$$

which we then cast as the first-order system

$$
\left(\begin{array}{c}
u_{x} \\
v_{x}
\end{array}\right)=\left(\begin{array}{c}
v \\
\omega \partial_{\tau} u+f(u)_{x}-c v
\end{array}\right)=\left(\begin{array}{c}
v \\
\omega \partial_{\tau} u+f_{u}(u) v-c v
\end{array}\right)
$$

We view (3.4) as an equation for $U=(u, v)$ in $Y=H^{1}\left(S^{1}\right) \times H^{1 / 2}\left(S^{1}\right)$ with $S^{1}=[0,2 \pi] / \sim$, and shall also use the space $X=L^{2}\left(S^{1}\right) \times L^{2}\left(S^{1}\right)$. The system (3.4) is invariant under the $S^{1}$-action

$$
\Gamma: S^{1} \longrightarrow L(Y, Y), \quad \sigma \longmapsto \Gamma_{\sigma}, \quad\left[\Gamma_{\sigma} U\right](x, \tau)=U(x, \tau-\sigma) .
$$

We record that the fixed-point space Fix $\Gamma \cong \mathbb{R}^{n} \times \mathbb{R}^{n}$ of this action consists precisely of all time-independent functions, and (3.4) restricted to Fix $\Gamma$ becomes the usual travelling-wave ODE

$$
\left(\begin{array}{c}
u_{x} \\
v_{x}
\end{array}\right)=\left(\begin{array}{c}
v \\
f_{u}(u) v-c v
\end{array}\right)
$$

which is equivalent to (2.3). Equation (3.6) possesses the equilibria $U_{\text {eq }}=(u, 0)$ for $u \in \mathbb{R}^{n}$ and the heteroclinic orbits $Q^{\varepsilon}(x):=\left(q^{\varepsilon}, q_{x}^{\varepsilon}\right)(x)$ for $c=c^{\varepsilon}: Q^{\varepsilon}(x)$ connects $U_{\mathrm{eq}}^{-}(\varepsilon)=\left(u_{-}^{\varepsilon}, 0\right)$ to $U_{\mathrm{eq}}^{+}(\varepsilon)=\left(u_{+}^{\varepsilon}, 0\right)$ with

$$
T_{Q^{\varepsilon}(x)} W_{-}^{\mathrm{u}}+T_{Q^{\varepsilon}(x)} W_{+}^{\mathrm{cs}}=\mathbb{R}^{2 n},
$$

where $W_{ \pm}^{j}:=W^{j}\left(U_{\text {eq }}^{ \pm}(\varepsilon)\right)$. The transversality of the intersection in (3.7) is a consequence of the following dimension count for $\varepsilon=0$ : Since $\operatorname{dim} W_{-}^{\mathrm{u}}=p+1$ and $\operatorname{dim} W_{+}^{\mathrm{cs}}=2 n-p$, it suffices to show that the only nontrivial solution in the intersection of the tangent spaces is $Q_{x}^{0}(x)$. This, in turn, is true because any 
nontrivial solution $(u, v)(x)$ of the variational equation of (3.6) which is exponentially localized at $x=-\infty$ gives a bounded solution $u(x)$ of the variational equation of (2.3). Any such solution is exponentially localized and must therefore, by assumption, be a multiple of $q_{x}^{0}(x)$.

Next, we linearize the full system (3.4) in the solution $Q^{0}=\left(q^{0}, q_{x}^{0}\right)$ for $\omega=\omega_{0}$ to get

$$
V_{x}=\left(\begin{array}{cc}
0 & 1 \\
\omega_{0} \partial_{\tau}+f_{u u}\left(q^{0}\right)\left[q_{x}^{0}, \cdot\right] & f_{u}\left(q^{0}\right)-c^{0}
\end{array}\right) V, \quad V \in Y .
$$

For $x \rightarrow \pm \infty$, we obtain the asymptotic systems

$$
V_{x}=\left(\begin{array}{cc}
0 & 1 \\
\omega_{0} \partial_{\tau} & f_{u}\left(u_{ \pm}^{0}\right)-c^{0}
\end{array}\right) V, \quad V \in Y
$$

whose properties we discuss first. Equations (3.8) and (3.9) leave each subspace $Y_{k}:=\left\{\mathrm{e}^{\mathrm{i} k \tau} \hat{V} ; \hat{V} \in \mathbb{C}^{2 n}\right\}$ invariant for $k \in \mathbb{Z}$. If we restrict (3.9) to $Y_{k}$, we obtain the $2 n \times 2 n$ system

$$
\hat{V}_{x}=\left(\begin{array}{cc}
0 & 1 \\
\mathrm{i} k \omega_{0} & f_{u}\left(u_{ \pm}^{0}\right)-c^{0}
\end{array}\right) \hat{V}, \quad \hat{V} \in \mathbb{C}^{2 n},
$$

where $V=\mathrm{e}^{\mathrm{i} k \tau} \hat{V}$. For $k \neq 0$, the matrices in (3.10) are hyperbolic: $\nu=\mathrm{i} \kappa$ is an eigenvalue if, and only if, $\operatorname{det}\left[-\kappa^{2}-\mathrm{i} \kappa\left(f_{u}\left(u_{ \pm}^{0}\right)-c^{0}\right)-\mathrm{i} k \omega_{0}\right]=0$, which is excluded since $f_{u}\left(u_{ \pm}^{0}\right)$ was assumed to have only real eigenvalues ${ }^{1}$. For $|k| \rightarrow \infty$, the eigenvalues of the matrices in (3.10) are

$$
\nu_{j}= \pm \sqrt{\mathrm{i} \omega_{0} k}\left(1+\mathrm{O}\left(|k|^{-1 / 2}\right)\right) \quad \text { with eigenfunction }\left(\begin{array}{c}
\nu_{j} e_{j} \\
e_{j}
\end{array}\right)
$$

where $e_{j}$ denotes the canonical basis in $\mathbb{R}^{n}$. In particular, the stable and unstable eigenspaces have a uniform angle in $H^{1}\left(S^{1}\right) \times H^{1 / 2}\left(S^{1}\right)$ as $|k| \rightarrow \infty$, and therefore for all $k \neq 0$; see also [12, Lemma 3.3]. Thus, we can apply the results in $[10,12]$ to conclude that $(3.9)$ restricted to $Y_{\mathrm{h}}:=\overline{\bigoplus_{k \neq 0} Y_{k}}$ has exponential dichotomies $\Phi_{ \pm, \mathrm{h}}^{\mathrm{s}, \mathrm{u}}(x, y)$ on $\mathbb{R}^{ \pm}$since the perturbation

$$
\left(\begin{array}{cc}
0 & 0 \\
f_{u u}\left(q^{0}(x)\right)\left[q_{x}^{0}(x), \cdot\right] & f_{u}\left(q^{0}(x)\right)-f_{u}\left(u_{ \pm}^{0}\right)
\end{array}\right): H^{1} \times H^{1 / 2} \longrightarrow H^{1} \times H^{1 / 2}
$$

is bounded independently of $x$ and converges to zero as $|x| \rightarrow \infty$. We define

$$
\begin{aligned}
\nu_{ \pm}^{\mathrm{s}} & :=-\frac{1}{2} \sup \left\{\operatorname{Re} \nu_{j} ; \operatorname{Re} \nu_{j}<0, \nu_{j} \text { is an eigenvalue of }(3.10)^{ \pm} \text {for some } k \in \mathbb{Z}\right\} \\
\nu_{ \pm}^{\mathrm{u}} & :=\frac{1}{2} \inf \left\{\operatorname{Re} \nu_{j} ; \operatorname{Re} \nu_{j}>0, \nu_{j} \text { is an eigenvalue of }(3.10)^{ \pm} \text {for some } k \in \mathbb{Z}\right\}
\end{aligned}
$$

and observe that $\nu_{ \pm}^{\mathrm{s}}, \nu_{ \pm}^{\mathrm{u}}>0$ due to (3.11). The spaces

$$
\begin{aligned}
& E_{+}^{\mathrm{cs}}=\left\{V_{0} \in Y ; \exists \text { solution } V(x) \text { of }(3.8) \text { on } \mathbb{R}^{+} \text {with } V(0)=V_{0}, \sup _{x \geq 0}|V(x)|<\infty\right\} \\
& E_{-}^{\mathrm{u}}=\left\{V_{0} \in Y ; \exists \text { solution } V(x) \text { of }(3.8) \text { on } \mathbb{R}^{-} \text {with } V(0)=V_{0}, \sup _{x \geq 0}|V(x)| \mathrm{e}^{\nu_{-}^{\mathrm{u}}|x|}<\infty\right\}
\end{aligned}
$$

are closed subspaces of $Y$.

Claim. We have

$$
\begin{aligned}
E_{+}^{\mathrm{cs}} \cap E_{-}^{\mathrm{u}} & =\mathbb{R} Q_{x}^{0}(0) \oplus \mathbb{R} V_{1}(0) \oplus \mathbb{R} V_{2}(0) \\
Y & =\left[E_{+}^{\mathrm{cs}}+E_{-}^{\mathrm{u}}\right] \oplus \mathbb{R} \Psi_{1}(0) \oplus \mathbb{R} \Psi_{2}(0)
\end{aligned}
$$

\footnotetext{
${ }^{1}$ Imaginary spatial eigenvalues $\nu=\mathrm{i} \kappa$ are actually equivalent to essential spectrum at $\lambda=\mathrm{i} \omega_{0} k$ so that, for more general viscosity matrices and fluxes, the analysis goes through provided the Hopf eigenvalue $i \omega_{0}$ is not resonant with essential spectrum on the imaginary axis.
} 
where, using the definitions of $v_{j}$ and $\psi_{j}$ from $\S 3.1$,

$$
V_{1}(x):=\cos \tau\left(\begin{array}{c}
v_{1} \\
\partial_{x} v_{1}
\end{array}\right)(x)+\sin \tau\left(\begin{array}{c}
v_{2} \\
\partial_{x} v_{2}
\end{array}\right)(x), \quad V_{2}(x):=-\sin \tau\left(\begin{array}{c}
v_{1} \\
\partial_{x} v_{1}
\end{array}\right)(x)+\cos \tau\left(\begin{array}{c}
v_{2} \\
\partial_{x} v_{2}
\end{array}\right)(x)
$$

and

$$
\Psi_{1}(x):=\cos \tau\left(\begin{array}{c}
\tilde{\psi}_{1} \\
\psi_{1}
\end{array}\right)(x)+\sin \tau\left(\begin{array}{c}
\tilde{\psi}_{2} \\
\psi_{2}
\end{array}\right)(x), \quad \Psi_{2}(x):=-\sin \tau\left(\begin{array}{l}
\tilde{\psi}_{1} \\
\psi_{1}
\end{array}\right)(x)+\cos \tau\left(\begin{array}{c}
\tilde{\psi}_{2} \\
\psi_{2}
\end{array}\right)(x)
$$

with $\tilde{\psi}_{j}:=-\partial_{x} \psi_{j}-\left[f_{u}^{T}\left(q^{0}\right)-c^{0}\right] \psi_{j}$ for $j=1,2$.

Proof. The characterization of $E_{+}^{\mathrm{cs}}$ and $E_{-}^{\mathrm{u}}$ is a consequence of the existence of exponential dichotomies on $Y_{\mathrm{h}}$ and the dynamics of the travelling-wave ODE (3.6). First, recall that the dynamics decouples, so that we can write

$$
E_{+}^{\mathrm{cs}} \bigoplus_{k \in \mathbb{Z}}\left(E_{+}^{\mathrm{cs}} \cap Y_{k}^{0}\right), \quad E_{-}^{\mathrm{u}}=\bigoplus_{k \in \mathbb{Z}}\left(E_{-}^{\mathrm{u}}+\cap Y_{k}^{0}\right)
$$

We know that the strong unstable manifold $W^{\mathrm{u}}\left(U_{\text {eq }}^{-}(0)\right)$ and the center-stable manifold $W^{\mathrm{cs}}\left(U_{\mathrm{eq}}^{+}(0)\right)$ of $(3.6)$ intersect transversely along $Q^{0}(x)$; see (3.7). Thus,

$$
\operatorname{span} Q_{x}^{0}(0)=E_{+}^{\mathrm{cs}} \cap E_{-}^{\mathrm{u}} \cap Y_{0} .
$$

Next, $V_{0} \in Y_{\mathrm{h}}$, the subspace of non-zero Fourier modes $k \neq 0$, lies in $E_{+}^{\mathrm{cs}} \cap E_{-}^{\mathrm{u}}$ if, and only if, $V(x)$ satisfies (3.9) on $\mathbb{R}$ with $V(x) \rightarrow 0$ exponentially as $|x| \rightarrow \infty$. Since (3.9) on $Y$ decouples, we find that such a solution can be taken in the form $V(x)=\mathrm{e}^{\mathrm{i} k \tau}\left(v, v_{x}\right)(x)$ for some integer $k \neq 0$. In particular, $v(x)$ satisfies

$$
\mathcal{L}^{0} v=\mathrm{i} k \omega_{0} v,
$$

and is therefore an $L^{2}$-eigenfunction of $\mathcal{L}^{0}$ to the eigenvalue $\lambda=\mathrm{i} k \omega_{0}$. Inspecting our hypotheses on $\mathcal{L}^{0}$, (3.12) follows. To prove (3.13), we consider the adjoint equation

$$
\Psi_{x}=-\left(\begin{array}{cc}
0 & -\mathrm{i} \omega_{0}+f_{u u}^{T}\left(q^{0}\right)\left[q_{x}^{0}, \cdot\right] \\
1 & f_{u}^{T}\left(q^{0}\right)-c^{0}
\end{array}\right) \Psi, \quad \Psi \in Y
$$

of (3.9), taken with respect to the inner product in the space $X=L^{2}\left(S^{1}\right) \times L^{2}\left(S^{1}\right)$. We note that the functions $\Psi_{j}(x)$ from (3.15) satisfy (3.16). A calculation shows that

$$
\frac{\mathrm{d}}{\mathrm{d} x}\langle V(x), \Psi(x)\rangle_{X}=0 \quad \text { for all } x \in \mathbb{R}
$$

for solutions $V(x)$ of $(3.9)$ and $\Psi(x)$ of (3.16); see [13] for similar arguments. Using the relation between $(3.16)$ and $\left[\mathcal{L}^{0}\right]^{*}$, we conclude that $(3.13)$ is met.

Note that the direction $Q_{x}^{0}(0) \in E_{+}^{\mathrm{cs}} \cap E_{-}^{\mathrm{u}}$ corresponds to the flow direction. To remove it, we shall later use the hyperplane

$$
\mathcal{S}:=\left[\mathbb{R} Q_{x}^{0}(0)\right]^{\perp} \subset Y .
$$

Next, we discuss the nonlinear equation (3.4) near the orbit $Q^{0}(x)$ for $\omega$ close to $\omega_{0}$ and $\varepsilon$ close to zero. It is convenient to set

$$
\omega=\omega_{0}+\Omega
$$

and to consider

$$
\left(\begin{array}{c}
u_{x} \\
v_{x}
\end{array}\right)=\left(\begin{array}{c}
v \\
\left(\omega_{0}+\Omega\right) \partial_{\tau} u+f_{u}(u) v-c^{\varepsilon} v
\end{array}\right)
$$

near the orbit $Q^{0}=\left(q^{0}, q_{x}^{0}\right)$ for $(\varepsilon, \Omega)$ close to zero. Using the fact that the linearized equation (3.8) can be solved using exponential dichotomies (whose existence we established above), we can proceed as in [12, 
$\S 3.5]$ and [20] to prove the existence of unstable and center-stable manifolds for (3.4) near the viscous shock. More precisely, there exist constants $\delta>0$ and $K>0$ such that

$$
\begin{aligned}
\mathcal{W}_{\varepsilon, \Omega}^{\mathrm{u}}:= & \left\{U_{0} \in Y ; \exists \text { solution } U(x) \text { of }(3.18) \text { on } \mathbb{R}^{-}: U(0)=U_{0},\left|U_{0}-Q^{0}(0)\right|<\delta,\right. \\
& \left.\left|U(x)-U_{\text {eq }}^{-}(\varepsilon)\right| \leq K \mathrm{e}^{-\nu_{-}^{\mathrm{u}}|x|} \text { for } x \leq 0\right\} \\
\mathcal{W}_{\varepsilon, \Omega}^{\mathrm{cs}}:= & \left\{U_{0} \in Y ; \exists \text { solution } U(x) \text { of }(3.18) \text { on } \mathbb{R}^{+}: U(0)=U_{0},\left|U_{0}-Q^{0}(0)\right|<\delta,\right. \\
& \left.\exists U_{\text {eq }}^{+} \in Y \text { with }\left|U_{\text {eq }}^{+}-U_{\text {eq }}^{+}(0)\right|<\delta \text { so that }\left|U(x)-U_{\text {eq }}^{+}\right| \leq K \mathrm{e}^{-\nu_{+}^{\mathrm{s}}|x|} \text { for } x \geq 0\right\}
\end{aligned}
$$

are smooth manifolds that are invariant under the action of the group $\Gamma$ defined in (3.5) and that depend smoothly on $(\varepsilon, \Omega)$ near zero. Moreover, $Q^{\varepsilon}(0) \in \mathcal{W}_{\varepsilon, \Omega}^{\mathrm{u}} \cap \mathcal{W}_{\varepsilon, \Omega}^{\mathrm{cs}}$, and the tangent spaces of the invariant manifolds at this point of intersection are given by

$$
T_{Q^{0}(0)} \mathcal{W}_{0,0}^{\mathrm{u}}=E_{-}^{\mathrm{u}}, \quad T_{Q^{0}(0)} \mathcal{W}_{0,0}^{\mathrm{cs}}=E_{+}^{\mathrm{cs}} .
$$

Note that the center-stable manifold $\mathcal{W}_{\varepsilon, \Omega}^{\text {cs }}$ is in effect given as the union of stable manifolds to the manifold $\left\{U_{\text {eq }}^{+}=(u, 0) ; u \in \mathbb{R}^{n}\right\}$ of asymptotic states, and therefore unique. Smoothness with respect to $\Omega$ can be shown by employing the coordinate change

$$
z=x \sqrt{\omega}, \quad(\tilde{u}, \tilde{v})=(u, v / \sqrt{\omega})
$$

which transforms (3.18) into an equation whose right-hand side depends smoothly on $\Omega$.

Finding solutions of (2.2), with temporal frequency $\omega$ near $\omega_{0}$, that converge asymptotically to constants as $x \rightarrow \pm \infty$, is therefore equivalent to finding elements $U_{0}$ in the intersection

$$
\mathcal{W}_{\varepsilon, \Omega}^{\mathrm{u}} \cap \mathcal{W}_{\varepsilon, \Omega}^{\mathrm{cs}} \cap\left[Q^{0}(0)+\mathcal{S}\right]
$$

for $\Omega$ close to zero, with $\mathcal{S}$ as in (3.17). Note that $U_{0}$ will have nontrivial time- $\tau$ dependence if, and only if, $U_{0}$ has a nonzero $Y_{\mathrm{h}}$-component. The minimal period will be $2 \pi / \omega$ if the component of $U_{0}$ in $Y_{1}$ does not vanish. We use Lyapunov-Schmidt reduction to determine the intersection (3.19). To this end, we write

$$
E_{+}^{\mathrm{cs}} \cap \mathcal{S}=\tilde{E}_{+}^{\mathrm{cs}} \oplus \operatorname{span}\left\{V_{1}(0), V_{2}(0)\right\}, \quad E_{-}^{\mathrm{u}} \cap \mathcal{S}=\tilde{E}_{-}^{\mathrm{u}} \oplus \operatorname{span}\left\{V_{1}(0), V_{2}(0)\right\} .
$$

There are then unique smooth maps

$$
\begin{array}{rc}
G^{\mathrm{cs}}(\cdot ; \varepsilon, \Omega): & \tilde{E}_{+}^{\mathrm{cs}} \oplus \operatorname{span}\left\{V_{1}(0), V_{2}(0)\right\} \longrightarrow \tilde{E}_{-}^{\mathrm{u}} \oplus \operatorname{span}\left\{\Psi_{1}(0), \Psi_{2}(0)\right\}, \\
G^{\mathrm{u}}(\cdot ; \varepsilon, \Omega): & \tilde{E}_{-}^{\mathrm{u}} \oplus \operatorname{span}\left\{V_{1}(0), V_{2}(0)\right\} \longrightarrow \tilde{E}_{+}^{\mathrm{cs}} \oplus \operatorname{span}\left\{\Psi_{1}(0), \Psi_{2}(0)\right\},
\end{array}
$$

with

$$
Q^{\varepsilon}(0)+\operatorname{graph} G^{j}(\cdot ; \varepsilon, \Omega)=\mathcal{W}_{\varepsilon, \Omega}^{j} \cap\left[Q^{0}(0)+\mathcal{S}\right], \quad j=\mathrm{cs}, \mathrm{u}
$$

and $D_{U} G^{j}(0 ; 0,0)=0$ for $j=\mathrm{cs}, \mathrm{u}$. In particular, both maps are equivariant under the $S^{1}$-action $\Gamma$. Thus, intersections of $\mathcal{W}_{\varepsilon, \Omega}^{\mathrm{u}}$ and $\mathcal{W}_{\varepsilon, \Omega}^{\mathrm{cs}}$ in $Q^{0}(0)+\mathcal{S}$ are in one-to-one correspondence with the roots of the mapping

$$
\begin{aligned}
G(\cdot ; \varepsilon, \Omega): \mathbb{R} \times \tilde{E}_{-}^{\mathrm{u}} \times \tilde{E}_{+}^{\mathrm{cs}} & \longrightarrow \tilde{E}_{-}^{\mathrm{u}} \oplus \tilde{E}_{+}^{\mathrm{cs}} \oplus \operatorname{span}\left\{\Psi_{1}(0), \Psi_{2}(0)\right\} \\
\left(a, w^{\mathrm{u}}, w^{\mathrm{cs}}\right) & \longmapsto w^{\mathrm{u}}+G^{\mathrm{u}}\left(w^{\mathrm{u}}+a V_{1}(0) ; \varepsilon, \Omega\right)-\left[w^{\mathrm{cs}}+G^{\mathrm{cs}}\left(w^{\mathrm{cs}}+a V_{1}(0) ; \varepsilon, \Omega\right)\right],
\end{aligned}
$$

where we factored out the nontrivial $S^{1}$-action on $\operatorname{span}\left\{V_{1}(0), V_{2}(0)\right\}$. Lyapunov-Schmidt reduction shows that there is a unique map

$$
W: \quad U_{\delta}(0) \subset \mathbb{R}^{3} \longrightarrow \tilde{E}_{-}^{\mathrm{u}} \times \tilde{E}_{+}^{\mathrm{cs}}, \quad(a, \varepsilon, \Omega) \longmapsto\left(W^{\mathrm{u}}(a, \varepsilon, \Omega), W^{\mathrm{cs}}(a, \varepsilon, \Omega)\right)
$$

so that

$$
G\left(a, w^{\mathrm{u}}, w^{\mathrm{cs}} ; \varepsilon, \Omega\right)=0 \quad \text { if, and only if, } \quad\left\langle\Psi_{j}(0), G(a, W(a, \varepsilon, \Omega) ; \varepsilon, \Omega)\right\rangle_{X}=0 \text { for } j=1,2 .
$$


Furthermore, $W$ is smooth in $(a, \varepsilon, \Omega)$ and we have $D_{(a, \varepsilon, \Omega)} W(0,0,0)=0$. In fact, since $G(0,0,0 ; \varepsilon, \Omega) \equiv 0$ due to $Q^{\varepsilon}(0) \in \mathcal{W}_{\varepsilon, \Omega}^{\mathrm{u}} \cap \mathcal{W}_{\varepsilon, \Omega}^{\mathrm{cs}}$ for all $(\varepsilon, \Omega)$, we have in addition that $W(0, \varepsilon, \Omega)=0$ for all small $(\varepsilon, \Omega)$, so that

$$
W(a, \varepsilon, \Omega)=a \mathrm{O}(|a|+|\varepsilon|+|\Omega|) .
$$

It suffices therefore to solve the reduced equations

$$
\left\langle\Psi_{j}(0), G(a, W(a, \varepsilon, \Omega) ; \varepsilon, \Omega)\right\rangle_{X}=0 \text { for } j=1,2 .
$$

To derive an expression for (3.21), we write (3.18) as

$$
U_{x}=F(U, \varepsilon, \Omega) .
$$

Using the coordinates $U=Q^{\varepsilon}+\tilde{U}$, we find that $\tilde{U}$ satisfies

$$
\tilde{U}_{x}=F_{U}\left(Q^{0}, 0,0\right) \tilde{U}+\mathcal{N}(\tilde{U}, \varepsilon, \Omega, x),
$$

where

$$
\mathcal{N}(\tilde{U}, \varepsilon, \Omega, x):=F\left(Q^{\varepsilon}+\tilde{U}, \varepsilon, \Omega\right)-F\left(Q^{\varepsilon}, \varepsilon, \Omega\right)-F_{U}\left(Q^{0}, 0,0\right) \tilde{U}=\mathrm{O}(|\tilde{U}|(|\tilde{U}|+|\varepsilon|+|\Omega|)) .
$$

Using the variation-of-constant formula that captures unstable and center-stable manifolds (see e.g. [20] or [12, Proposition 3.13]) and the fact that $\Psi_{j}(x)$ satisfies (3.16) together with [13, Lemma 5.1], we find that (3.21) is given by

$$
\int_{-\infty}^{\infty}\left\langle\Psi_{j}(x), \mathcal{N}\left(\tilde{U}^{ \pm}(x), \varepsilon, \Omega, x\right)\right\rangle_{X} \mathrm{~d} x=0, \quad j=1,2
$$

where $\tilde{U}^{ \pm}(x)$ satisfies $(3.23)$ on $\mathbb{R}^{ \pm}$with $\tilde{U}^{-}(0)=a V_{1}(0)+W^{\mathrm{u}}(a, \varepsilon, \Omega)$ and $\tilde{U}^{+}(0)=a V_{1}(0)+W^{\mathrm{cs}}(a, \varepsilon, \Omega)$. If we write (3.25) as $\Pi(a, \varepsilon, \Omega)=0$, then we know from the preceding discussion that $\Pi(0, \varepsilon, \Omega)=0$ for all $(\varepsilon, \Omega)$ : this solution corresponds to the persisting Lax shocks in Fix $\Gamma$. To obtain genuinely time-periodic solutions corresponding to $a \neq 0$, we write

$$
\Pi(a, \varepsilon, \Omega)=a \tilde{\Pi}(a, \varepsilon, \Omega)
$$

and consider $\tilde{\Pi}(a, \varepsilon, \Omega)=0$, which can be solved by the implicit function theorem provided the $2 \times 2$ matrix $D_{(\varepsilon, \Omega)} \tilde{\Pi}(0,0,0)$ is invertible. Equation (3.26) shows that

$$
D_{(\varepsilon, \Omega)} \tilde{\Pi}(0,0,0)=D_{a} D_{(\varepsilon, \Omega)} \Pi(0,0,0) .
$$

It therefore suffices to prove that

$$
D_{(\varepsilon, \Omega)} \tilde{\Pi}(0,0,0)=\left[\left.D_{(\varepsilon, \Omega)} \int_{-\infty}^{\infty}\left\langle\Psi_{j}(x), \mathcal{N}\left(\tilde{U}^{ \pm}(x), \varepsilon, \Omega, x\right)\right\rangle_{X} \mathrm{~d} x\right|_{(\varepsilon, \Omega)=0}\right]_{j=1,2}
$$

is invertible. We know that

$$
\tilde{U}^{+}(x)=a V_{1}(x)+W^{\mathrm{cs}}(a, \varepsilon, \Omega)(x), \quad \tilde{U}^{-}(x)=a V_{1}(x)+W^{\mathrm{u}}(a, \varepsilon, \Omega)(x)
$$

which we rewrite as

$$
\tilde{U}^{ \pm}(x)=a\left[V_{1}(x)+\tilde{W}^{ \pm}(x ; a, \varepsilon, \Omega)\right]
$$

with

$$
\begin{array}{lll}
\tilde{W}^{-}(x ; a, \varepsilon, \Omega) & :=\frac{1}{a} W^{\mathrm{u}}(a, \varepsilon, \Omega)(x)=\mathrm{O}(|a|+|\varepsilon|+|\Omega|), & x \in \mathbb{R}^{-} \\
\tilde{W}^{+}(x ; a, \varepsilon, \Omega) & :=\frac{1}{a} W^{\mathrm{cs}}(a, \varepsilon, \Omega)(x)=\mathrm{O}(|a|+|\varepsilon|+|\Omega|), & x \in \mathbb{R}^{+}
\end{array}
$$


due to the estimate (3.20). Thus,

$$
\left.\frac{\mathrm{d}^{2}}{\mathrm{~d}(\varepsilon, \Omega) \mathrm{d} a} \mathcal{N}\left(\tilde{U}^{ \pm}(x), \varepsilon, \Omega, x\right)\right|_{(a, \varepsilon, \Omega)=0}=\left.D_{(\varepsilon, \Omega)} F_{U}\left(Q^{\varepsilon}(x), \varepsilon, \Omega\right)\right|_{(a, \varepsilon, \Omega)=0} V_{1}(x) .
$$

Upon comparing (3.22) with (3.18), we see that

$$
\begin{aligned}
\left.\frac{\mathrm{d}^{2}}{\mathrm{~d} \Omega \mathrm{d} a} \mathcal{N}\right|_{(a, \varepsilon, \Omega)=0} & =\left(\begin{array}{cc}
0 & 0 \\
\partial_{\tau} & 0
\end{array}\right) V_{1} \\
\left.\frac{\mathrm{d}^{2}}{\mathrm{~d} \varepsilon \mathrm{d} a} \mathcal{N}\right|_{(a, \varepsilon, \Omega)=0} & =\left(\begin{array}{cc}
0 & 0 \\
\left.\partial_{\varepsilon}\left(f_{u u}\left(q^{\varepsilon}\right)\left[q_{x}^{\varepsilon}, \cdot\right]\right)\right|_{\varepsilon=0} & \left.\partial_{\varepsilon}\left[f_{u}\left(q^{\varepsilon}\right)-c^{\varepsilon}\right]\right|_{\varepsilon=0}
\end{array}\right) V_{1} .
\end{aligned}
$$

Substituting the expressions (3.14) and (3.15) for $V_{1}$ and $\Psi_{j}$, and using the normalization (3.1), we obtain

$$
\left[\left.\frac{\mathrm{d}^{2}}{\mathrm{~d} \Omega \mathrm{d} a} \int_{-\infty}^{\infty}\left\langle\Psi_{j}(x), \mathcal{N}\left(\tilde{U}^{ \pm}(x), \varepsilon, \Omega, x\right)\right\rangle_{X} \mathrm{~d} x\right|_{(\varepsilon, \Omega)=0}\right]_{j=1,2}=\left(\begin{array}{c}
0 \\
1
\end{array}\right) \in \mathbb{R}^{2} .
$$

An analogous computation for the derivative with respect to $\varepsilon$ gives

$$
\begin{aligned}
{\left[\left.\frac{\mathrm{d}^{2}}{\mathrm{~d} \varepsilon \mathrm{d} a} \int_{-\infty}^{\infty}\left\langle\Psi_{1}(x), \mathcal{N}\left(\tilde{U}^{ \pm}(x), \varepsilon, \Omega, x\right)\right\rangle_{X} \mathrm{~d} x\right|_{(\varepsilon, \Omega)=0}\right] } & =\left\langle\psi_{1}, \partial_{\varepsilon} \partial_{x}\left[f_{u}\left(q^{\varepsilon}\right) v_{1}-c^{\varepsilon} v_{1}\right]_{\varepsilon=0}\right\rangle_{L^{2}} \\
& =-\left\langle\psi_{1},\left.\partial_{\varepsilon} \mathcal{L}^{\varepsilon}\right|_{\varepsilon=0} v_{1}\right\rangle_{L^{2}} \\
& =-\operatorname{Re} \lambda_{\varepsilon}(0)
\end{aligned}
$$

where we used (3.3) to obtain the last step. Hence, we find that the Jacobian in (3.27) is given by

$$
D_{(\varepsilon, \Omega)} \tilde{\Pi}(0,0,0)=\left(\begin{array}{cc}
-\operatorname{Re} \lambda_{\varepsilon}(0) & 0 \\
\star & 1
\end{array}\right)
$$

which is invertible due to our hypothesis on the transverse crossing of the Hopf eigenvalues.

Upon applying the implicit function theorem to solve $\tilde{\Pi}(a, \varepsilon, \Omega)=0$ we see that there exist unique functions $\left(\varepsilon_{*}, \Omega_{*}\right)(a) \in \mathbb{R}^{2}$ and $Q^{*}(0 ; a) \in Y$, defined for $|a|<\delta$, so that

$$
Q^{*}(0 ; a) \in \mathcal{W}_{\left(\varepsilon_{*}, \Omega_{*}\right)(a)}^{\mathrm{u}} \cap \mathcal{W}_{\left(\varepsilon_{*}, \Omega_{*}\right)(a)}^{\mathrm{cs}} \cap\left[Q^{\varepsilon}(0)+\mathcal{S}\right] .
$$

These functions are smooth and satisfy $\left.\partial_{a}\left(\varepsilon_{*}, \Omega_{*}\right)\right|_{a=0}=0, Q^{*}(0 ; 0)=Q^{0}(0)$, and

$$
Q^{*}(x ; a)=Q^{\varepsilon_{*}(a)}(x)+a\left[V_{1}(x)+\tilde{W}^{ \pm}\left(x ; a, \epsilon_{*}(a), \Omega_{*}(a)\right)\right]=: Q^{\varepsilon_{*}(a)}(x)+a \tilde{Q}^{*}(x ; a) .
$$

By construction, we have $Q^{*}(x ; a) \rightarrow U_{\text {eq }}^{-}\left(\varepsilon_{*}(a)\right)$ as $x \rightarrow-\infty$. Furthermore, $Q^{*}(x ; a) \in \mathcal{W}_{\left(\varepsilon_{*}, \Omega_{*}\right)(a)}^{\text {cs }}$ from which we infer that there exists a $U_{*}^{+}(a) \in \mathbb{R}^{n}$ with $\left|U_{*}^{+}(a)-U_{\mathrm{eq}}^{+}\left(\varepsilon_{*}(a)\right)\right|<\delta$, so that $Q^{*}(x ; a) \rightarrow U_{*}^{+}(a)$ exponentially as $x \rightarrow \infty$ with rate $\nu_{+}^{\mathrm{s}}$. We claim that $U_{*}^{+}(a)=U_{\text {eq }}^{+}\left(\varepsilon_{*}(a)\right)$. To prove this claim, consider the smooth functional

$$
\mathcal{E}: \quad Y \longrightarrow \mathbb{R}^{n}, \quad(u, v) \longmapsto \int_{0}^{2 \pi}[v-f(u)+c u] \mathrm{d} \tau .
$$

This functional is conserved under the evolution of (3.4): If $U(x)=(u, v)(x) \in Y$ is a solution of (3.4), then $v=u_{x}$ and

$$
\frac{\mathrm{d}}{\mathrm{d} x} \mathcal{E}(U(x))=\frac{\mathrm{d}}{\mathrm{d} x} \int_{0}^{2 \pi}[v-f(u)+c u] \mathrm{d} \tau=\int_{0}^{2 \pi}\left[v_{x}-f_{u}(u) v+c v\right] \mathrm{d} \tau \stackrel{(3.4)}{=} \int_{0}^{2 \pi} \omega u_{\tau} \mathrm{d} \tau=0
$$

since $u$ is $2 \pi$-periodic in $\tau$. Furthermore, for $U=\left(u_{0}, v_{0}\right) \in \operatorname{Fix} \Gamma \subset Y$, we have

$$
D_{U} \mathcal{E}\left(u_{0}, v_{0}\right)\left(\begin{array}{c}
\tilde{u} \\
\tilde{v}
\end{array}\right)=\int_{0}^{2 \pi}\left[\tilde{v}-f_{u}\left(u_{0}\right) \tilde{u}+c \tilde{u}\right] \mathrm{d} \tau=\tilde{v}_{0}-f_{u}\left(u_{0}\right) \tilde{u}_{0}+c \tilde{u}_{0} \in \mathbb{R}^{n} .
$$


In particular, $D_{U} \mathcal{E}\left(u_{ \pm}^{0}, 0\right)$ restricted to $\mathbb{R}^{n} \times\{0\} \subset Y_{0}$ is invertible, since we assumed that none of the characteristic speeds vanishes in the frame that moves with speed $c^{0}$. Since $Q^{\varepsilon_{*}(a)}(x)$ connects $U_{\text {eq }}^{-}\left(\varepsilon_{*}(a)\right)$ to $U_{\text {eq }}^{+}\left(\varepsilon_{*}(a)\right)$, they have the same $\mathcal{E}$-values, and the preceding argument shows that there is no other equilibrium $U_{\text {eq }}^{+}$near $U_{\text {eq }}^{+}\left(\varepsilon_{*}(a)\right)$ with the $\mathcal{E}$-value of $U_{\text {eq }}^{+}\left(\varepsilon_{*}(a)\right)$. Therefore, $Q^{*}(x ; a) \rightarrow U_{\text {eq }}^{ \pm}\left(\varepsilon_{*}(a)\right)$ for $x \rightarrow \pm \infty$.

This completes the existence proof of the bifurcating oscillatory viscous shock waves. The uniqueness statement in Theorem 1 is a consequence of our construction which captures all solutions that lie in the intersection of $\mathcal{W}_{\varepsilon, \Omega}^{\mathrm{u}}$ and $\mathcal{W}_{\varepsilon, \Omega}^{\mathrm{cs}}$. We remark that (3.34) also shows that any time-periodic localized travelling viscous shock wave satisfies the Rankine-Hugoniot condition (2.4).

To prepare the ground for the following spectral stability proof, we derive an expression for $\varepsilon_{a a}(0)$. We set $(\varepsilon, \Omega)=0$ and compute the derivatives

$$
\frac{\mathrm{d}^{j}}{\mathrm{~d} a^{j}} \Pi_{i}(a, 0,0)=\frac{\mathrm{d}^{j}}{\mathrm{~d} a^{j}} \int_{-\infty}^{\infty}\left\langle\Psi_{i}(x), \mathcal{N}\left(\tilde{U}^{ \pm}(x), 0,0, x\right)\right\rangle_{X} \mathrm{~d} x
$$

at $a=0$ for $i=1,2$. Using the expressions (3.24) for $\mathcal{N}$ and (3.28) for $\tilde{U}^{ \pm}$together with the estimate (3.29) for $\tilde{W}(x ; a, 0,0)$, we easily find that the second derivatives vanish at $a=0$ for $i=1,2$, while

$$
\begin{aligned}
\kappa_{3} & :=\frac{\mathrm{d}^{3}}{\mathrm{~d} a^{3}} \Pi_{1}(a, 0,0) \\
& =\int_{-\infty}^{\infty}\left\langle\Psi_{1}(x), F_{U U U}\left(Q^{0}(x), 0,0\right)\left[V_{1}(x)\right]^{3}+3 F_{U U}\left(Q^{0}(x), 0,0\right)\left[V_{1}(x), \tilde{W}_{a}(x ; 0,0,0)\right]\right\rangle_{X} \mathrm{~d} x .
\end{aligned}
$$

A straightforward calculation using (3.31) then shows that

$$
\varepsilon_{a a}(0)=\frac{\kappa_{3}}{3 \operatorname{Re} \lambda_{\varepsilon}(0)} .
$$

\section{Stability of the bifurcating modulated viscous shocks}

This section is devoted to the proof of Theorem 2. Our goal is to determine the Floquet spectrum

$$
\Sigma=\left\{\lambda \in \mathbb{C} ; \mathrm{e}^{2 \pi \lambda} \in \text { spectrum of } \Phi_{2 \pi}\right\}
$$

associated with the evolution $\Phi_{t}$ of the linearization

$$
\omega v_{\tau}=\partial_{x}\left[v_{x}+c v-f_{u}\left(q^{*}(x, \tau ; a)\right) v\right]
$$

of (2.2) about $q^{*}$ on $\mathcal{C}_{\text {unif }}^{0}$. Note that $(\varepsilon, \omega)$ and $q^{*}$ depend smoothly on the parameter $a$ introduced in $\S 3$, and so do $c=c^{\varepsilon}$ and the asymptotic rest states $u_{ \pm}^{\varepsilon}$ through $\varepsilon=\varepsilon_{*}(a)$; we will suppress this dependence for most of the proof.

The Floquet spectrum $\Sigma$ is the disjoint union of the essential spectrum $\Sigma_{\text {ess }}$ and the point spectrum $\Sigma_{\mathrm{pt}}$, which consists, by definition, of all isolated eigenvalues with finite multiplicity. Since the modulated shock $q^{*}(x, \tau ; a)$ converges exponentially to the constants $u_{ \pm}^{\varepsilon}$ as $x \rightarrow \pm \infty$, uniformly in $\tau$, the set $\Sigma_{\text {ess }}$ is bounded to the right by the essential spectra

$$
\Sigma_{\text {ess }}^{ \pm}=\left\{\lambda \in \mathbb{C} ; \operatorname{det}\left(k^{2}+\mathrm{i} k\left[f_{u}\left(u_{ \pm}^{\varepsilon}\right)-c^{\varepsilon}\right]+\lambda\right)=0 \text { for some } k \in \mathbb{R}\right\}
$$

of $u_{ \pm}^{\varepsilon}$ (see, for instance, [13, Proposition 2.10]), which touch the imaginary axis at $\lambda=0$ and lie otherwise in the open left half-plane due to our hypothesis that the eigenvalues of $f_{u}\left(u_{ \pm}^{\varepsilon}\right)$ are real and simple.

It therefore suffices to locate point spectrum, that is, isolated Floquet exponents $\lambda$ which are captured, via the ansatz $v(x, \tau)=\mathrm{e}^{\lambda \tau} u(x, \tau)$ with $u(x, \tau+2 \pi)=u(x, \tau)$ for all $\tau$, by the equation

$$
\omega_{*}(a) u_{\tau}+\lambda u=\partial_{x}\left[u_{x}+c^{\varepsilon_{*}(a)} u-f_{u}\left(q^{*}(x, \tau ; a)\right) u\right]
$$




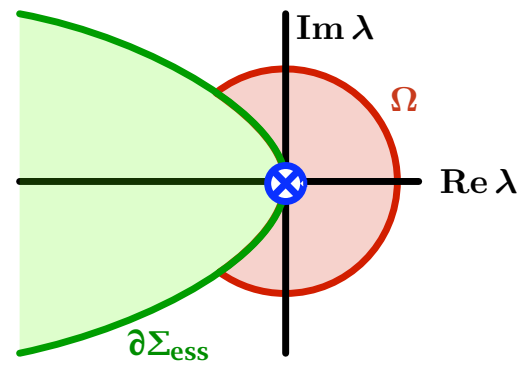

Figure 2: The definition of the open set $\Omega \subset \mathbb{C}$ in the complex Floquet plane is shown. The embedded Floquet exponent at the origin has multiplicity at least equal to two with eigenfunctions $q_{x}^{*}$ and $q_{\tau}^{*}$.

or, alternatively, by

$$
\begin{aligned}
V_{x} & =\left(\begin{array}{cc}
0 & 1 \\
\omega \partial_{\tau}+\lambda+f_{u u}\left(q^{*}\right)\left[q_{x}^{*}, \cdot\right] & f_{u}\left(q^{*}\right)-c
\end{array}\right) V \\
& =\left[F_{U}\left(Q^{*}(x ; a), \epsilon_{*}(a), \Omega_{*}(a)\right)+\lambda \mathcal{B}\right] V, \quad V \in Y
\end{aligned}
$$

with $Q^{*}(x ; a)=\left(q^{*}, q_{x}^{*}\right)(x, \cdot ; a)$ from $(3.32)$.

Since we assumed spectral stability for $\epsilon=0$ except for the Hopf eigenvalues and the translational eigenvalue at the origin (which all contribute to the Floquet exponent $\lambda=0$ ), it suffices to find all isolated Floquet exponents of (4.1) in a fixed small neighbourhood of the origin. We choose an open set $\Omega \subset \mathbb{C}$ as indicated in Figure 2. Standard theory implies that $\lambda \in \Omega$ is a Floquet exponent if, and only if, (4.1) has a nontrivial exponentially decaying solution on $\mathbb{R}$. Taking the limit $x \rightarrow \pm \infty$ in (4.1), we obtain the asymptotic operators

$$
\left(\begin{array}{cc}
0 & 1 \\
\omega \partial_{\tau}+\lambda & f_{u}\left(u_{ \pm}\right)-c
\end{array}\right)
$$

We denote the eigenvectors and eigenvalues of $\left[f_{u}\left(u_{ \pm}\right)-c\right]$ by $r_{j}^{ \pm}$and $\nu_{j}^{ \pm}$, respectively. As discussed in $\S 3$, the operators in (4.2) are hyperbolic for $\lambda=0$ except for the $n$-fold eigenvalue $\mathcal{V}=0$ with eigenvectors $\left(r_{j}^{ \pm}, 0\right) \in Y_{0}$. This eigenvalue and the associated eigenvectors become

$$
\mathcal{V}_{j}^{ \pm}=-\frac{\lambda}{\nu_{j}^{ \pm}}+\mathrm{O}\left(\lambda^{2}\right), \quad R_{j}^{ \pm}=\left(\begin{array}{c}
r_{j}^{ \pm} \\
\mathcal{V}_{j}^{ \pm} r_{j}^{ \pm}
\end{array}\right), \quad j=1, \ldots, n
$$

for $\lambda$ near zero. For $\lambda \in \Omega$, the unstable eigenspace $E_{-}^{\infty}(\lambda, a)$ at $x=-\infty$ and the stable eigenspace $E_{+}^{\infty}(\lambda, a)$ at $x=\infty$ are therefore given by

$$
E_{+}^{\infty}:=E_{+}^{\mathrm{s}} \oplus \mathcal{R}_{+}, \quad E_{-}^{\infty}:=E_{-}^{\mathrm{u}} \oplus \mathcal{R}_{-}, \quad \mathcal{R}_{ \pm}=\operatorname{span}\left\{R_{j}^{ \pm} ; \nu_{j}^{ \pm} \gtrless 0\right\},
$$

and these spaces depend smoothly on $a$ and are analytic in $\lambda$ for $\lambda$ near zero. Note that $\operatorname{dim} \mathcal{R}^{+}=p$ and $\operatorname{dim} \mathcal{R}^{-}=n-p-1$ with $p$ as in $\S 2$.

Lemma 4.1 There are unique closed subspaces $E_{ \pm}(\lambda, a)$ of $Y$, defined and analytic in $\lambda$ near zero and smooth in $a \geq 0$, such that $V(x)$ is a bounded solution of (4.1) on $\mathbb{R}^{ \pm}$for some $\lambda \in \Omega$ if, and only if, $V(0) \in E_{ \pm}(\lambda, a)$.

Proof. We begin by considering (4.1) with $Q^{*}$ replaced by $Q^{\varepsilon}$. In this case, equation (4.1) decouples on each Fourier space $Y_{k}$, and the claimed statement holds for $Q^{\varepsilon}$ due to the Gap Lemma [5,9] applied in $Y_{0}$ and exponential-dichotomy theory together with estimates as in [12, Lemma 3.3] in the other Fourier spaces. Since the difference of $Q^{\varepsilon}$ and $Q^{*}$ is small for all $x$ and decays to zero exponentially as $|x| \rightarrow \infty$, these results carry over to (4.1) using, for instance, the integral formulation in $[11,(4.12)$ in $\S 4.3]$; see also $[17, \S 7.6]$ for a slightly different proof. 
Lemma 4.1 shows that Floquet exponents in $\Omega$ can be found by seeking nontrivial intersections of $E_{-}(\lambda, a)$ and $E_{+}(\lambda, a)$. To determine their intersections, we first set $(\lambda, a)=0$ to see what these spaces look like at onset and then use perturbation theory to analyse the case when $(\lambda, a) \neq 0$.

Hence, let $\lambda=0$, then (4.1) is simply the variational equation

$$
V_{x}=F_{U}\left(Q^{*}(x ; a), \epsilon_{*}(a), \Omega_{*}(a)\right) V, \quad V \in Y
$$

of the modulated wave $Q^{*}$. When $a=0$, we have $Q^{*}=Q^{0}$, and (4.4) describes Floquet exponents at $\lambda=0$ of the unperturbed viscous shock $q^{0}$. In particular, (4.4) decouples on each Fourier space $Y_{k}$, and our hypotheses on the Evans function and the spectral properties of the viscous shock imply that

$$
\begin{aligned}
E_{+}(0,0) \cap E_{-}(0,0) & =\operatorname{span}\left\{Q_{x}^{0}(0), V_{1}(0), V_{2}(0)\right\} \\
{\left[E_{+}(0,0)+E_{-}(0,0)\right]^{\perp} } & =\operatorname{span}\left\{\Psi_{0}, \Psi_{1}(0), \Psi_{2}(0)\right\}
\end{aligned}
$$

for an appropriate nonzero vector $\Psi_{0} \in Y_{0}$. Next, consider (4.4) for an arbitrary a near zero. First, note that the gradient of the $j$ th component of the conserved quantity $\mathcal{E}$ from (3.33), computed in the $X=L^{2}\left(S^{1}\right) \times L^{2}\left(S^{1}\right)$ scalar product, is given by

$$
\nabla\left\langle\mathcal{E}(u, v), e_{j}\right\rangle_{\mathbb{R}^{n}}=\left(\begin{array}{c}
-\left[f_{u}^{T}(u)-c\right] e_{j} \\
e_{j}
\end{array}\right), \quad j=1, \ldots, n,
$$

where $e_{j}$ denotes the canonical basis in $\mathbb{R}^{n}$. Our analysis of $\mathcal{E}$ in $\S 3$ implies that these $n$ gradients are linearly independent for $a=0$, and we therefore have $\operatorname{dim} E^{*}(a)=n$ for all small $a$ where

$$
E^{*}(a):=\operatorname{span}\left\{\nabla\left\langle\mathcal{E}\left(Q^{*}(0 ; a)\right), e_{j}\right\rangle_{\mathbb{R}^{n}} ; j=1, \ldots, n\right\} .
$$

The gradients in (4.6) also satisfy the adjoint equation of (4.4), again computed in $X$, which shows that

$$
\frac{\mathrm{d}}{\mathrm{d} x}\left\langle\nabla\left\langle\mathcal{E}\left(Q^{*}(x ; a)\right), e_{j}\right\rangle_{\mathbb{R}^{n}}, V(x)\right\rangle_{X} \equiv 0 \quad j=1, \ldots, n
$$

for each solution $V(x)$ of (4.4); this can also be verified directly by evaluating (4.7). We denote by $\ell_{j}^{ \pm}(a)$ the smooth eigenvectors of $\left[f_{u}^{T}\left(u_{ \pm}^{\varepsilon}\right)-c^{\varepsilon}\right]$ at $\varepsilon_{*}(a)$ associated with the eigenvalues $\nu_{j}^{ \pm}$, and define

$$
E_{ \pm}^{*}(a)=\operatorname{span}\left\{\nabla\left\langle\mathcal{E}\left(Q^{*}(0 ; a)\right), \ell_{j}^{ \pm}(a)\right\rangle_{\mathbb{R}^{n}} ; \nu_{j}^{ \pm} \lessgtr 0\right\} \subset E^{*}(a) .
$$

Set $a=0$, then $\operatorname{dim} E_{+}^{*}(0)=n-p$ and $\operatorname{dim} E_{-}^{*}(0)=p+1$. Equations (4.3), (4.6) and (4.7) imply that $E_{ \pm}^{*}(0) \perp_{X} E_{ \pm}(0,0)$ and, in fact, that $E_{ \pm}^{*}(0)$ are perpendicular to each solution of (4.4) at $a=0$ that decays exponentially at $x=-\infty$ or $x=\infty$. Equation (4.5) implies then that $E_{+}^{*}(0) \cap E_{-}^{*}(0)=\operatorname{span}\left\{\Psi_{0}\right\}$, and therefore $\operatorname{dim}\left[E_{+}^{*}(0)+E_{-}^{*}(0)\right]=n$. Since $E_{ \pm}^{*}(a) \subset E^{*}(a)$ for all $a$, and the latter space is $n$-dimensional for all $a$, we have $E_{+}^{*}(a) \pitchfork E_{-}^{*}(a)$ in $E^{*}(a)$, and the dimensions of the sum and intersection of $E_{ \pm}^{*}(a)$ cannot change for $a$ close to zero. Hence, we can choose a nonzero basis vector $\Psi_{0}^{*}(0 ; a)$ in the one-dimensional intersection $E_{+}^{*}(a) \cap E_{-}^{*}(a)$ that depends smoothly on $a$ as well as linearly independent smooth elements $\Psi_{j}^{ \pm}(0 ; a) \in E_{ \pm}^{*}(a)$, with $j=1, \ldots, n-p-1$ for the $+\operatorname{sign}$ and $j=1, \ldots, p$ for the - sign, so that $\Psi_{j}^{ \pm}(0 ; a) \perp_{X} \Psi_{0}^{*}(0 ; a)$ for all $j$. Using (4.3), (4.6) and (4.8), we see that

$$
\Psi_{j}^{ \pm}(0 ; a) \perp_{X} E_{ \pm}(0, a), \quad \Psi_{0}^{*}(0 ; a) \perp_{X}\left[E_{+}(0, a)+E_{-}(0, a)\right]
$$

for all $a$. Lastly, we define

$$
\Psi_{1,2}^{*}(0 ; a):=[1-P(a)] \Psi_{1,2}(0 ; a)
$$

where $P(a)$ is the orthogonal projection in $X$ onto $E_{+}^{*}(a)+E_{-}^{*}(a)$.

Having prepared the ground for the forthcoming analysis, we now return to the full eigenvalue problem (4.1)

$$
V_{x}=\left[F_{U}\left(Q^{*}(x ; a), \epsilon_{*}(a), \Omega_{*}(a)\right)+\lambda \mathcal{B}\right] V .
$$


We seek solutions $V^{ \pm}(x)$ on $\mathbb{R}^{ \pm}$of the form

$$
V^{ \pm}(x)=b_{0} Q_{x}^{*}(x ; a)+b_{1} V_{1}(x)+b_{2} \tilde{Q}_{\tau}^{*}(x ; a)+\tilde{V}^{ \pm}(x ; \lambda, a) b
$$

with $b=\left(b_{0}, b_{1}, b_{2}\right), Q^{*}=Q^{\varepsilon}+a \tilde{Q}^{*}$ as in (3.32), and

$$
\tilde{V}^{ \pm}(0 ; \lambda, a) b \perp \operatorname{span}\left\{Q_{x}^{*}(0 ; a), V_{1}(0), \tilde{Q}_{\tau}^{*}(0 ; a)\right\},
$$

for all $b$, so that

$$
\begin{aligned}
& V^{+}(0)-V^{-}(0) \in \operatorname{span}\left\{\Psi_{j}^{*}(0 ; a) ; j=0,1,2\right\} \\
& \operatorname{dist}\left(\frac{1}{\left|V^{ \pm}(x)\right|_{X}} V^{ \pm}(x), E_{ \pm}^{\infty}(\lambda, a)\right) \rightarrow 0 \text { as } x \rightarrow \pm \infty .
\end{aligned}
$$

Using exponential dichotomies as in $\S 3$, we can then easily show that (4.1) and (4.11)-(4.14) has unique solutions for each $b \in \mathbb{R}^{3}$ and $(\lambda, a)$ near zero and that these solutions depend analytically on $\lambda$ and smoothly on $a$. In particular, $E_{+}(\lambda, a) \cap E_{-}(\lambda, a) \neq\{0\}$ if, and only if, $\operatorname{det} \mathcal{D}(\lambda, a)=0$ where

$$
\mathcal{D}(\lambda, a): \quad \mathbb{R}^{3} \longrightarrow \mathbb{R}^{3}, \quad b \longmapsto \mathcal{D}(\lambda, a) b=\left(\left\langle\Psi_{j}^{*}(0 ; a), V^{+}(0)-V^{-}(0)\right\rangle_{X}\right)_{j=0,1,2} .
$$

We will now compute the Taylor expansion of $\mathcal{D}$ to solve $\operatorname{det} \mathcal{D}(\lambda, a)=0$.

First, we set $a=0$ so that $Q^{*}=Q^{0}, Q_{x}^{0}=: V_{0}$ and $\tilde{Q}_{\tau}^{*}=V_{2}$. A calculation similar to the derivation of (3.25) gives

$$
\partial_{\lambda} \mathcal{D}(0,0)=\left(\int_{\mathbb{R}}\left\langle\Psi_{i}^{*}(x ; 0), \mathcal{B} V_{j}(x)\right\rangle_{X} \mathrm{~d} x\right)_{i, j=0,1,2}=\operatorname{diag}\left(\int_{\mathbb{R}}\left\langle\Psi_{j}(x), \mathcal{B} V_{j}(x)\right\rangle_{X} \mathrm{~d} x\right)=\operatorname{diag}\left(M_{0}, 1,1\right),
$$

where we used the normalization (3.1). Our hypothesis that $\lambda=0$ is a simple root of the Evans function of the viscous shock at $\varepsilon=0$ implies that $M_{0} \neq 0$.

Next, we set $\lambda=0$ and compute derivatives with respect to $a$. Since $\lambda=0$, the eigenvalue problem reduces to the variational equation (4.4). In particular, both $\partial_{x} Q^{*}(x ; a)$ and $\partial_{\tau} \tilde{Q}^{*}(x ; a)$ are solutions of (4.4) that satisfy (4.14), and we can set $b_{0}=b_{2}=0$ as they make no contribution to $\mathcal{D}(0, a)$. We focus therefore on $V^{ \pm}(x)=V_{1}(x)+\tilde{V}^{ \pm}(x ; 0, a)$ for which (4.9) and (4.14) together imply

$$
\left\langle\Psi_{0}^{*}(0 ; a), V^{+}(0)-V^{-}(0)\right\rangle_{X}=0
$$

for all $a$. The equation for $\tilde{V}$ is

$$
\tilde{V}_{x}=F_{U}\left(Q^{0}(x), 0,0\right) \tilde{V}+\left[F_{U}\left(Q^{*}(x ; a), \varepsilon_{*}(a), \Omega_{*}(a)\right)-F_{U}\left(Q^{0}(x), 0,0\right)\right]\left(V_{1}(x)+\tilde{V}\right)
$$

and, proceeding as before and using (3.32), we obtain

$$
\left.\frac{\mathrm{d}}{\mathrm{d} a}\left\langle\Psi_{j}^{*}(0 ; a), \tilde{V}^{+}(0 ; 0, a)-\tilde{V}^{-}(0 ; 0, a)\right\rangle_{X}\right|_{a=0}=\int_{\mathbb{R}}\left\langle\Psi_{j}(x), F_{U U}\left(Q^{0}(x), 0,0\right)\left[V_{1}(x), V_{1}(x)\right]\right\rangle_{X} \mathrm{~d} x
$$

for $j=1,2$. Inspecting (3.14) and (3.15), we see that the integrands vanish pointwise for each $x$. Summarizing the findings obtained so far, we have

$$
\mathcal{D}(\lambda, a)=\left(\begin{array}{ccc}
M_{0} \lambda & 0 & 0 \\
0 & \lambda+\mathrm{O}\left(a^{2}\right) & 0 \\
0 & \mathrm{O}\left(a^{2}\right) & \lambda
\end{array}\right)+\mathrm{O}(|\lambda|(|\lambda+| a \mid)) .
$$

Thus, it remains to compute the diagonal $\mathrm{O}\left(a^{2}\right)$ term. Expanding (4.16), we see that the second derivative with respect to $a$ of this diagonal term is given by

$$
\begin{aligned}
\partial_{a a} \mathcal{D}_{22}(0,0)= & \int_{-\infty}^{\infty}\left\langle\Psi_{1}(x), F_{U U U}\left(Q^{0}(x), 0,0\right)\left[V_{1}(x)\right]^{3}+3 F_{U U}\left(Q^{0}(x), 0,0\right)\left[V_{1}(x), \partial_{a} \tilde{V}(x ; 0,0)\right]\right. \\
& \left.+\left.\varepsilon_{a a}(0) D_{\varepsilon}\left(F_{U}\left(Q^{\varepsilon}(x), \varepsilon, 0\right)\right)\right|_{\varepsilon=0} V_{1}(x)+\Omega_{a a}(0) \partial_{\Omega} F_{U}\left(Q^{0}(x), 0,0\right) V_{1}\right\rangle_{X} \mathrm{~d} x \\
= & \int_{-\infty}^{\infty}\left\langle\Psi_{1}(x), F_{U U U}\left(Q^{0}(x), 0,0\right)\left[V_{1}(x)\right]^{3}+3 F_{U U}\left(Q^{0}(x), 0,0\right)\left[V_{1}(x), \partial_{a} \tilde{V}(x ; 0,0)\right]\right\rangle_{X} \mathrm{~d} x \\
& -\varepsilon_{a a}(0) \operatorname{Re} \lambda_{\varepsilon}(0)
\end{aligned}
$$


where the term involving $\Omega$ vanishes for the same reason that shows that the first component in (3.30) is zero. Comparing the integral term in the above expression with (3.35), we see that they coincide provided $\partial_{a} \tilde{V}(x ; 0,0)=\partial_{a} \tilde{W}(x ; 0,0,0)$. The following lemma, whose proof we postpone until after we finished the discussion of $\mathcal{D}(\lambda, a)$, states that this identity indeed holds.

Lemma 4.2 We have $\partial_{a} \tilde{V}(x ; 0,0)=\partial_{a} \tilde{W}(x ; 0,0,0)$.

Thus, we can conclude that

$$
\partial_{a a} \mathcal{D}_{22}(0,0)=\kappa_{3}-\varepsilon_{a a}(0) \operatorname{Re} \lambda_{\varepsilon}(0) \stackrel{(3.36)}{=} 2 \varepsilon_{a a}(0) \operatorname{Re} \lambda_{\varepsilon}(0)
$$

and consequently

$$
\mathcal{D}(\lambda, a)=\left(\begin{array}{ccc}
M_{0} \lambda & 0 & 0 \\
0 & \lambda+\varepsilon_{a a}(0) \operatorname{Re} \lambda_{\varepsilon}(0) a^{2}+\mathrm{O}\left(a^{3}\right) & 0 \\
0 & \mathrm{O}\left(a^{2}\right) & \lambda
\end{array}\right)+\mathrm{O}(|\lambda|(|\lambda+| a \mid)) .
$$

The equation $\operatorname{det} \mathcal{D}(\lambda, a)=0$ has therefore precisely three solutions, counted with multiplicity, given by $\lambda=0$ with multiplicity two and a simple root at

$$
\lambda_{*}(a)=-\varepsilon_{a a}(0) \operatorname{Re} \lambda_{\varepsilon}(0) a^{2}+\mathrm{O}\left(a^{3}\right),
$$

so that $\lambda_{*}(a)$ and $\varepsilon_{*}(a)$ have opposite signs since we assumed that $\operatorname{Re} \lambda_{\varepsilon}(0)>0$. Subject to establishing Lemma 4.2, this completes the proof of Theorem 2.

Proof of Lemma 4.2. Expanding the relevant equations for $\tilde{V}$ and $\tilde{W}$, we find that both $\partial_{a} \tilde{V}^{ \pm}(x ; 0,0)$ and $\partial_{a} \tilde{W}^{ \pm}(x ; 0,0,0)$ satisfy the linear inhomogeneous differential equation

$$
V_{x}=F_{U}\left(Q^{0}(x), 0,0\right) V+F_{U U}\left(Q^{0}(x), 0,0\right)\left[V_{1}(x), V_{1}(x)\right] .
$$

The asymptotic boundary conditions for the hyperbolic directions coincide for both functions, but differ for the center directions. We shall show that the center components of $\partial_{a} \tilde{V}^{ \pm}(0 ; 0,0)$ and $\partial_{a} \tilde{W}^{ \pm}(0 ; 0,0,0)$ are equal to each other from which we can infer that the two solutions coincide as claimed.

We begin by discussing $\tilde{V}^{ \pm}(x ; 0,0)$. Equation (4.15) implies that the $\Psi_{0}^{*}(0 ; a)$ components of $\tilde{V}^{ \pm}(0 ; 0, a)$ coincide for all $a$. Since $\Psi_{j}^{ \pm}(0 ; a)$ is perpendicular to the space on the right-hand side of (4.12), we also have

$$
\left\langle\Psi_{j}^{ \pm}(0 ; a), \tilde{V}^{+}(0 ; 0, a)-\tilde{V}^{-}(0 ; 0, a)\right\rangle_{X}=0
$$

for all $j$ and all $a$, and we conclude that the center components of $\tilde{V}^{ \pm}(0 ; 0, a)$ coincide for all $a$. Since $V^{ \pm}(0) \in E_{ \pm}(0, a)$ for all $a$, equation (4.9) gives

$$
\left\langle\Psi_{j}^{ \pm}(0 ; a), V^{ \pm}(0)\right\rangle_{X}=0, \quad\left\langle\Psi_{0}^{*}(0 ; a), V^{ \pm}(0)\right\rangle_{X}=0
$$

for all $a$, and a Taylor expansion gives

$$
\begin{aligned}
& \left\langle\partial_{a} \Psi_{j}^{ \pm}(0 ; 0), V_{1}(0)\right\rangle_{X}+\left\langle\Psi_{j}^{ \pm}(0 ; 0), \partial_{a} \tilde{V}^{ \pm}(0 ; 0,0)\right\rangle_{X}=0 \quad \forall j \\
& \left\langle\partial_{a} \Psi_{0}^{*}(0 ; 0), V_{1}(0)\right\rangle_{X}+\left\langle\Psi_{0}^{*}(0 ; 0), \partial_{a} \tilde{V}^{ \pm}(0 ; 0,0)\right\rangle_{X}=0 .
\end{aligned}
$$

We now turn to $\partial_{a} \tilde{W}^{ \pm}(0 ; 0,0,0)$. We set $(\varepsilon, \Omega)=0$ and consider the solution pieces $U^{ \pm}(x)=Q^{0}(x)+$ $a\left[V_{1}(x)+\tilde{W}^{ \pm}(x ; a, 0,0)\right]$ from $\S 3$. By construction, we have $U^{ \pm}(x) \in \mathcal{W}_{0,0}^{\mathrm{u}}$, and the conserved quantity $\mathcal{E}\left(U^{-}(x)\right)$ does therefore not depend on $a$. Its derivative with respect to $a$ is given by

$$
\begin{aligned}
0 & =\frac{\mathrm{d}}{\mathrm{d} a} \mathcal{E}\left(Q^{0}(0)+a\left[V_{1}(0)+\tilde{W}^{-}(0 ; a, 0,0)\right]\right) \\
& =\left\langle\nabla \mathcal{E}\left(Q^{0}(0)+a\left[V_{1}(0)+\tilde{W}^{-}(0 ; a, 0,0)\right]\right), V_{1}(0)+\tilde{W}(0 ; a, 0,0)\right\rangle_{X} \\
& =\left\langle\nabla \mathcal{E}\left(Q^{0}(0)+a\left[V_{1}(0)+\mathrm{O}(a)\right]\right), V_{1}(0)+a \tilde{W}_{a}^{-}(0 ; 0,0,0)+\mathrm{O}\left(a^{2}\right)\right\rangle_{X} \\
& =a\left[\left\langle\left.\frac{\mathrm{d}}{\mathrm{d} a} \nabla \mathcal{E}\left(Q^{0}(0)+a V_{1}(0)\right)\right|_{a=0}, V_{1}(0)\right\rangle_{X}+\left\langle\nabla \mathcal{E}\left(Q^{0}(0)\right), \tilde{W}_{a}^{-}(0 ; 0,0,0)\right\rangle_{X}\right]+\mathrm{O}\left(a^{2}\right) .
\end{aligned}
$$


Thus, we get

$$
\begin{aligned}
\left\langle\partial_{a} \Psi_{j}^{ \pm}(0 ; 0), V_{1}(0)\right\rangle_{X}+\left\langle\Psi_{j}^{ \pm}(0 ; 0), \tilde{W}_{a}^{-}(0 ; 0,0,0)\right\rangle_{X} & =0 \\
\left\langle\partial_{a} \Psi_{0}^{*}(0 ; 0), V_{1}(0)\right\rangle_{X}+\left\langle\Psi_{0}^{*}(0 ; 0), \tilde{W}_{a}^{-}(0 ; 0,0,0)\right\rangle_{X} & =0 .
\end{aligned}
$$

We can proceed as before to show continuity of $\tilde{W}_{a}^{ \pm}(0 ; 0,0,0)$ in the center components. This fact, together with the continuity of $\tilde{V}_{a}(0 ; 0,0)$ in the center directions and equations (4.17) and (4.18), shows that the center components of $\partial_{a} \tilde{V}^{ \pm}(0 ; 0,0)$ and $\partial_{a} \tilde{W}^{ \pm}(0 ; 0,0,0)$ are indeed equal to each other as claimed.

\section{Discussion}

There are numerous possible generalizations and extensions of our results. The crucial ingredient is the existence of the evolution operators $\Phi_{ \pm}^{\mathrm{s}, \mathrm{u}}$ for the spatial dynamical system, which requires some hyperbolicity in the spatial dynamics. The results are clearly not dependent on the particular form of the viscosity matrix: nonlinear viscosity $B(u) u_{x x}$ is allowed as long as the essential spectrum is non-resonant with the Hopf eigenvalue (uniform positivity is typically sufficient). We can also allow parameter dependent fluxes: the parameter $\varepsilon$ may appear explicitly in the viscosity matrix and the flux $f=f(u ; \varepsilon)$.

Under- and overcompressive shocks can be treated similarly. All viscous shocks can be viewed as heteroclinic orbits in the second-order travelling-wave ODE

$$
u_{x x}+[c u-f(u)]_{x}=0
$$

which connect families of equilibria at $x= \pm \infty$. To set up the problem, we can, for instance, prescribe the values of $u$ on ingoing characteristics: Choose subsets $\mathcal{S}_{ \pm}$so that $T_{u_{ \pm}^{0}} \mathcal{S}_{ \pm} \oplus \mathcal{I}_{ \pm}=\mathbb{R}^{n}$, where $\mathcal{I}_{ \pm}$is the eigenspace belonging to eigenvalues $\nu^{ \pm} \lessgtr 0$ of $f_{u}\left(u_{ \pm}^{0}\right)$. We then seek viscous shock waves in the intersection of $\mathcal{W}^{\mathrm{u}}\left(\mathcal{S}_{-}\right)$and $\mathcal{W}^{\mathrm{s}}\left(\mathcal{S}_{+}\right)$. Both manifolds are $n$-dimensional, and we will then assume that their intersection along the viscous shock is transverse ${ }^{2}$ in the parameter $c$. One can now vary $\mathcal{S}_{ \pm}=\mathcal{S}_{ \pm}^{\varepsilon}$ and continue the transverse intersection provided the speed $c=c^{\varepsilon}$ is adjusted appropriately. If a pair of complex eigenvalues crosses the imaginary axis at $\varepsilon=0$, the analysis in this paper can be adapted easily to show that there is a unique family of oscillatory under- or overcompressive shocks bifurcating from the primary viscous shock.

The analysis extends also to the case of degenerate shock waves, where we allow for an additional center direction within the travelling-wave ODE in Fix $\Gamma$ at either $u_{-}^{0}$ or $u_{+}^{0}$. Again, suitable transversality conditions on the intersections of $\mathcal{W}_{-}^{\mathrm{u}}$ and $\mathcal{W}_{+}^{\mathrm{s}}$ together with appropriate assumptions on the nonlinear behaviour of the zero characteristic speed near the shock are needed.

Problems posed in infinite cylinders,

$$
u_{t}=\Delta u+\sum_{j} \partial_{x_{j}} f_{j}(u), \quad x \in \mathbb{R} \times \Omega
$$

for bounded cross-sections $\Omega \subset \mathbb{R}^{N}$ and with Neumann boundary conditions on $\mathbb{R} \times \partial \Omega$, say, can also be treated. The existence of exponential dichotomies for this problem follows from [10, 13].

The major open problem that we did not address in this paper is nonlinear stability of the bifurcating oscillatory viscous shocks. It should be possible to establish nonlinear stability using a combination of the approach via pointwise estimates developed by Howard and Zumbrun in [8, 21] and our spatial-dynamics technique which can be used to obtain the necessary estimates for the Green's function; this will be pursued elsewhere.

\footnotetext{
${ }^{2}$ This is equivalent to the assumption that $\lambda=0$ is a simple root of the Evans function associated with the PDE linearization at the shock.
} 


\section{References}

[1] T. Brand, M. Kunze, G. Schneider, and T. Seelbach. Hopf bifurcation and exchange of stability in diffusive media. Arch. Ration. Mech. Anal. 171 (2004) 263-296.

[2] S.-N. Chow and J.K. Hale. Methods of bifurcation theory. Springer, New York, 1982.

[3] A. Doelman, B. Sandstede, A. Scheel, and G. Schneider. The dynamics of modulated wave trains. Memoirs Amer. Math. Soc. (to appear).

[4] B. Fiedler, B. Sandstede, A. Scheel, and C. Wulff. Bifurcation from relative equilibria of noncompact group actions: skew products, meanders, and drifts. Documenta Mathematica 1 (1996) 479-505.

[5] R.A. Gardner and K. Zumbrun. The gap lemma and geometric criteria for instability of viscous shock profiles. Comm. Pure Appl. Math. 51 (1998) 797-855.

[6] M. Golubitsky, V. LeBlanc, and I. Melbourne. Meandering of the spiral tip - an alternative approach. J. Nonl. Sci. 7 (1997) 557-586.

[7] M. Haragus and A. Scheel. Corner defects in almost planar interface propagation. Ann. Inst. H. Poincaré Anal. Non Linéaire 23 (2006) 283-329.

[8] P. Howard and K. Zumbrun. Stability of undercompressive shock profiles. J. Diff. Eqns. 225 (2006) 308-360.

[9] T. Kapitula and B. Sandstede. Stability of bright solitary wave solutions to perturbed nonlinear Schrödinger equations. Physica D 124 (1998) 58-103.

[10] D. Peterhof, B. Sandstede, and A. Scheel. Exponential dichotomies for solitary wave solutions of semilinear elliptic equations on infinite cylinders. J. Diff. Eqns. 140 (1997) 266-308.

[11] B. Sandstede. Stability of travelling waves. In: Handbook of Dynamical Systems II (B Fiedler, ed.). North-Holland (2002) 983-1055.

[12] B. Sandstede and A. Scheel. Essential instability of pulses and bifurcations to modulated travelling waves. Proc. Roy. Soc. Edinburgh A 129 (1999) 1263-1290.

[13] B. Sandstede and A. Scheel. On the structure of spectra of modulated travelling waves. Math. Nachr. 232 (2001) 39-93.

[14] B. Sandstede and A. Scheel. Essential instabilities of fronts: bifurcation and bifurcation failure. Dynamical Systems 16 (2001) 1-28.

[15] B. Sandstede and A. Scheel Absolute instabilities of standing pulses. Nonlinearity 18 (2005) 331-378.

[16] B. Sandstede, A. Scheel, and C. Wulff. Dynamics of spiral waves on unbounded domains using centermanifold reduction. J. Diff. Eqns. 141 (1997) 122-149.

[17] A. Scheel. Coarsening fronts. Arch. Ration. Mech. Anal. 181 (2006) 505-534.

[18] B. Texier and K. Zumbrun. Relative Poincare-Hopf bifurcation and galloping instability of travelling waves. Preprint, 2005.

[19] B. Texier and K. Zumbrun. Galloping instability of viscous shock waves. Preprint, 2006.

[20] A. Vanderbauwhede and G. Iooss. Center manifold theory in infinite dimensions. Dynamics Reported 1 (1992) 125-163.

[21] K. Zumbrun and P. Howard. Pointwise semigroup methods and stability of viscous shock waves. Indiana Univ. Math. J. 47 (1998) 741-871. 